\title{
Avaliação do Sistema de Vigilância Sanitária do Sangue em âmbito federal, Brasil, 2007
}

\author{
Evaluation of the System of Sanitary Vigilance of Blood \\ at the federal level, Brazil, 2007
}

Daniel Marques Mota ${ }^{1}$

Daniel Roberto Coradi Freitas ${ }^{2}$

Wildo Navegantes de Araújo ${ }^{3}$

${ }^{1}$ Coordenação do Sistema Nacional de Gerenciamento de Produtos Controlados, Núcleo de Gestão do Sistema Nacional de Notificação e Investigação em Vigilância Sanitária, Agência Nacional de Vigilância Sanitária. Setor de Indústria e

Abastecimento (SIA) Trecho 5, Área Especial 57, Lote 200 . Bloco D, $1^{\circ}$ andar, Sala NUVIG, 71205-050

Brasília DF.

dmarques2003@yahoo.com.br

${ }^{2}$ Gerência de Tecidos,

Células e Órgãos, Gerência Geral de Sangue, Outros Tecidos, Células e Órgãos, Agência Nacional de Vigilância Sanitária

${ }^{3}$ Programa de Treinamento em Epidemiologia Aplicada aos Serviços do SUS

(Episus), Centro de Informações Estratégicas e Respostas em Vigilância em Saúde (CIEVS),

Departamento de Vigilância de Doenças Transmissíveis, Secretaria de Vigilância em Saúde, Ministério da Saúde
Abstract This study evaluated the Sanitary Vigilance System for Blood at federal government level (VIGISAN). This assessment included the following components: managerial system of registration of hemotherapeutic services; external evaluation program of quality of hemotherapy services; hemovigilance system; standardization and inspection of health services for blood and its components. Besides the description and the calculation of efficiency (cost-effectiveness ratio) of VIGISAN, the assessment consisted of two phases based on three methods. VIGISAN had a poorly defined structure, though it operated smoothly and its utility was acceptable for public health. From a total of four defined indicators to evaluate performance, one obtained a satisfactory result. Three out of the seven evaluation attributes achieved positive assessment in this study. The cost-effectiveness ratio was US\$37,965.16 per activity performed, which resulted in loss of efficiency for the system. This study demonstrates the importance of conducting evaluations of vigilance systems in public health, which should be performed periodically in order to contribute to improvement of the system and thus improve public health in the country.

Key words Performance evaluation, Blood, Brazilian System of Sanitary Vigilance, Sanitary vigilance
Resumo Este estudo consistiu em avaliar o Sistema de Vigilância Sanitária do Sangue em âmbito federal (Vigisan). Trata-se de um estudo de avaliação em saúde que contemplou os seguintes componentes selecionados do Vigisan: sistema gerencial de cadastro de serviços hemoterápicos, programa de avaliação externa da qualidade de serviços de hemoterapia, sistema de hemovigilância, normatização e inspeção dos serviços de sangue e seus componentes. Além da descrição e do cálculo da eficiência (razão custo-efetividade) do Vigisan, a avaliação foi composta de duas fases com base em três métodos. O Vigisan apresentou uma estrutura mal definida, funcionamento regular e utilidade aceitável para a saúde pública. De um total de quatro indicadores definidos para a avaliação de desempenho, um obteve resultado satisfatório. Dos sete atributos estudados, três alcançaram uma avaliação positiva. A razão custo-efetividade foi de $R \$ 80.865,80$ por atividade executada, o que resultou em perda de eficiência para o Sistema. Este estudo demonstra a importância da realização de avaliações de Sistemas de Vigilância em Saúde Pública, as quais devem ser realizadas periodicamente com a finalidade de contribuir no aperfeiçoamento do Sistema e, consequentemente, melhorar a saúde pública do país.

Palavras-chave Avaliação de desempenho, Sangue, Sistema Nacional de Vigilância Sanitária, Vigilância sanitária 


\section{Introdução}

A transfusão de sangue e seus componentes é um procedimento que apresenta risco de transmissão de doenças infecciosas e eventos adversos transfusionais ${ }^{1-4}$. Entre 5 e $10 \%$ das infecções pelo vírus HIV no mundo são transmitidos por transfusões de sangue e pelo uso de produtos sanguíneos contaminados. Além disso, muito mais receptores desses insumos são infectados pela hepatite B e C, sífilis e doença de Chagas ${ }^{1}$. O risco de eventos adversos transfusionais é estimado em 1:16.500 transfusões sanguíneas realizadas, sendo que a ocorrência de óbitos como resultado de um componente do sangue transfundido incorretamente é de aproximadamente $1: 1.500 .000^{2}$. Salienta-se que no Brasil ainda não é conhecido, com precisão, o número de transfusões realizadas. Dados projetados para 2007 apontam para a realização de quase três milhões de transfusões realizadas e de 9.000 reações transfusionais esperadas ${ }^{4}$.

A Organização Mundial da Saúde (OMS, 1999) recomenda às autoridades de saúde de todos os países a assumirem a responsabilidade na garantia do suprimento seguro e adequado de sangue à população ${ }^{1}$. Para tanto, a seleção adequada de doadores, o uso de testes de triagem efetivos, a manutenção de um sistema de hemovigilância e a elaboração de legislação e regulamentos técnicos dirigidos aos serviços de produção e transfusão de sangue e seus componentes são pré-requisitos para o uso seguro e eficaz desses insumos essenciais à vida ${ }^{1,5}$.

No Brasil, a vigilância sanitária (VISA) tem um papel primordial no controle do risco e da qualidade do sangue e seus componentes por intermédio de ações como normatização, inspeção sanitária, capacitação e educação sanitária, notificação e monitoramento de eventos adversos, licenciamento de estabelecimentos hemoterápicos e avaliação da qualidade de serviços de hemoterapia. O conjunto destas ações é desenvolvido por órgãos que compõem a VISA em nível federal, estadual e municipal e que integram o Sistema Nacional de Vigilância Sanitária (SNVS).

O exercício de VISA é uma função essencial na consolidação do Sistema Único de Saúde (SUS), que durante muito tempo no Brasil permaneceu como um campo à margem da Saúde Pública, esquecido quanto à sua importância para a saúde da população ${ }^{6}$. Após esse tempo, essa prática de vigilância foi resgatada pela Constituição Federal de 1988, Art. 200, II onde se prevê que compete ao SUS: "executar as ações de vigi- lância sanitária e epidemiológica bem como as de saúde do trabalhador"7.

Na prática de VISA existem problemas repetitivos de gestão, conflitos de interesses entre atores sociais, e muitas vezes, fragilidade organizacional e insuficiência da estrutura institucional para resolvê-los. Essas dificuldades, postas em um segundo plano no âmbito da VISA, reforçam uma convicção quanto à importância da avaliação para o processo de planejamento e gestão, podendo, também dar respostas quanto à adequação entre a execução das ações e a eliminação, diminuição ou prevenção de riscos de produtos e serviços sujeitos à VISA ${ }^{8}$.

No âmbito federal, a Agência Nacional de Vigilância Sanitária (Anvisa) é o órgão responsável pelo controle, redução e eliminação dos riscos sanitários com o uso de sangue e seus componentes, bem como pela produção, análise e difusão de informações sobre eventos adversos relacionados com esses produtos.

O objetivo deste estudo foi avaliar o Sistema de Vigilância Sanitária do Sangue em âmbito federal, denominado de Vigisan, em 2007, a partir de uma análise de componentes previamente estabelecidos e de responsabilidade de setores da Anvisa. Como todo sistema de vigilância em saúde pública, o Vigisan deve estar sujeito a um processo de avaliação, essencial para refletir sobre a eficiência das suas ações e sua importância para a saúde pública do país.

\section{Métodos}

Realizou-se um estudo de avaliação em saúde com foco na avaliação de sistemas de vigilância em saúde pública, cujas unidades de análise foram a Gerência de Sangue e Componentes (GESAC), a Gerência-Geral de Sangue, Outros Tecidos, Células e Órgãos (GGSTO) e a Unidade de Bio e Hemovigilância (UBHEM) da Anvisa. Os componentes do Vigisan avaliados foram: sistema gerencial de cadastro de serviços hemoterápicos (Hemocad), programa de avaliação externa da qualidade de serviços de hemoterapia (AEQ) em imuno-hematologia e sorologia, sistema de hemovigilância (SH), normatização e inspeção sanitária. A coleta dos dados ocorreu entre março e maio de 2008.

\section{Avaliação do Sistema}

A avaliação foi composta de duas fases: i) Geral, que incluiu a avaliação de desempenho do 
Sistema; e ii) Específica por atributos. A primeira foi obtida a partir de dados coletados na avaliação do AEQ imuno-hematologia e sorologia e $\mathrm{SH}$, cujo instrumento utilizado foi adaptado da metodologia de Moliner e Ochoa'. A avaliação de desempenho foi fundamentada no instrumento de Função Essencial de Saúde Pública - FESP no 6, proposto pela Organização Pan-americana de Saúde (OPAS) ${ }^{10,11}$. A avaliação específica foi baseada nas diretrizes de Avaliação de Sistemas de Vigilância em Saúde Pública do Centers for Disease Control and Prevention dos Estados Unidos (CDC/EUA) ${ }^{12}$. Além disso, conforme previsto nessas diretrizes, foi realizado uma descrição geral do Vigisan.

A avaliação dos componentes AEQ e SH foi baseada em informações referidas por gestores, técnicos e consultores das unidades de análise que preencheram questionários autoaplicáveis sobre a tríade estrutura-processo-resultado e cujo resultado correspondeu ao valor médio das respostas. No Quadro 1 está apresentada a forma de avaliação e interpretação dos aspectos mensurados.

A avaliação de desempenho foi também baseada em informações referidas por gestores e técnicos da GESAC e GGSTO que responderam ao questionário autoaplicável da FESP no 6, o qual mediu a capacidade institucional de normatização e inspeção sanitária. Os resultados fo- ram classificados como: baixo (0 a 45\%), regular (46 a 74\%), ótimo desejável (75 a 90\%) ou excelente (>90\%).

$\mathrm{Na}$ avaliação específica, os atributos mensurados foram: simplicidade, flexibilidade, aceitabilidade, representatividade, estabilidade, oportunidade e qualidade dos dados (Quadro 2).

A utilidade do sistema foi avaliada pela capacidade de cumprir seus objetivos a partir da inferência dos resultados obtidos na avaliação dos componentes estudados e de detectar, entre outros aspectos, um aumento de eventos adversos relacionados com o uso de sangue e componentes.

A avaliação da eficiência do Vigisan foi medida em termos de custo-efetividade e expressa em reais $(\mathrm{R} \$)$ por atividade executada. Os custos diretos mensurados foram: i) atividades programadas no orçamento de 2007 para as unidades de análise relacionadas com os componentes avaliados; e ii) recursos humanos. Os custos das atividades foram valorizados a preços que constavam no documento orçamentário enquanto que os custos com pessoal foi baseada na renda mensal, somado ao $13^{\circ}$ salário e a $1 / 3$ de férias. Aos recursos que fizeram parte da análise foram atribuídos valores em reais $(\mathrm{R} \$$ ) referentes a janeiro de 2007 (US\$ $1.00=\mathrm{R} \$ 2,13$ ).

O cálculo da efetividade foi baseado no número de atividades executadas dividido pela quantidade programada. Foi atribuída uma pontua-

Quadro 1. Avaliação dos aspectos: estrutura, processo e resultados do Vigisan. Brasil, 2007.

\begin{tabular}{|c|c|c|c|}
\hline Aspecto & Itens avaliados & Pontuação & Interpretação \\
\hline \multirow[t]{3}{*}{ Estrutura } & \multirow{3}{*}{$\begin{array}{l}\text { Organização } \\
\text { Recursos materiais e financeiros } \\
\text {. Recursos humanos } \\
\text {. Disponibilidade de informações para } \\
\text { a comunidade usuária de sangue }\end{array}$} & 0 a 43 & Estrutura mal definida \\
\hline & & 44 a 74 & Estrutura com imprecisões \\
\hline & & 75 a 85 & Estrutura adequadamente definida \\
\hline \multirow[t]{3}{*}{ Processo } & \multirow{3}{*}{$\begin{array}{l}\text { Cumprimento dos atributos } \\
\text { simplicidade, aceitabilidade, } \\
\text { flexibilidade, integralidade, } \\
\text { oportunidade, capacidade de } \\
\text { autorresposta, representatividade e } \\
\text { custos }\end{array}$} & 0 a 86 & Funcionamento deficiente \\
\hline & & 87 a 151 & Funcionamento regular \\
\hline & & 152 a 172 & Bom funcionamento \\
\hline \multirow[t]{3}{*}{ Resultados } & \multirow{3}{*}{$\begin{array}{l}\text { Utilidade do Sistema em aportar } \\
\text { informações para diferentes atores } \\
\text { Capacidade de: i) contribuir na } \\
\text { prevenção e controle de agravos; ii) } \\
\text { estimular a investigação } \\
\text { epidemiológico-sanitária; e iii) ajudar } \\
\text { no aperfeiçoamento da prática de VISA }\end{array}$} & 0 a 24 & Não útil \\
\hline & & 25 a 42 & Utilidade aceitável \\
\hline & & 43 a 48 & Muito útil \\
\hline
\end{tabular}


Quadro 2. Avaliação específica dos atributos do Vigisan. Brasil, 2007.

\begin{tabular}{|c|c|c|}
\hline Atributo & Forma de verificação & Pontuação/Classificação \\
\hline Simplicidade & $\begin{array}{l}\text { Quantidade excessiva no AEQ-Imuno- } \\
\text { hematologia de: i) dados coletados; ii) fonte de } \\
\text { dados; iii) documentos a serem elaborados; iv) } \\
\text { tempo dedicado às atividades do programa; e v) } \\
\text { organizações envolvidas. Outras perguntas } \\
\text { referiram-se a complexidade das tarefas e sobre } \\
\text { aspectos que afetam a dinâmica do programa, } \\
\text { como: i) duplicidade de dados; ii) coleta de dados } \\
\text { que não são analisados ou utilizados; e iii) tempo } \\
\text { elevado gasto na execução de tarefas. }\end{array}$ & $\begin{array}{l}\text { Pontuação: } 0 \text { a } 12 \\
\text { Classificação: simples }(\geq 10) \\
\text { ou complexa }(<10)\end{array}$ \\
\hline Flexibilidade & $\begin{array}{l}\text { Duas situações: i) transferência das atividades de } \\
\text { hemovigilância da GESAC para UBHEM e reflexo } \\
\text { no custo da operação do SH; e ii) observou-se, de } \\
\text { forma retrospectiva, como o Vigisan respondeu a } \\
\text { uma nova demanda para o sistema, o uso da auto- } \\
\text { hemoterapia por segmentos da população } \\
\text { brasileira. }\end{array}$ & $\begin{array}{l}\text { Pontuação: julgamento subjetivo } \\
\text { Classificação: flexível ou não } \\
\text { flexível. }\end{array}$ \\
\hline Aceitabilidade & $\begin{array}{l}\text { Relação entre o número de hospitais sentinelas } \\
\text { que apresentou plano de melhoria em } \\
\text { hemovigilância e o total de hospitais sentinelas } \\
\text { que receberam recursos financeiros da Anvisa e } \\
\text { participavam do projeto. }\end{array}$ & $\begin{array}{l}\text { Pontuação: } 0 \text { a } 100 \% \\
\text { Classificação: alta }(\geq 85 \%) \\
\text { ou baixa }(<85 \%)\end{array}$ \\
\hline Representatividade & $\begin{array}{l}\text { Percentual de serviços de hemoterapia cadastrados } \\
\text { no Hemocad por unidade da federação. O } \\
\text { Cadastro Nacional de Estabelecimentos de Saúde } \\
\text { (CNES) foi o padrão-ouro. }\end{array}$ & $\begin{array}{l}\text { Pontuação: } 0 \text { a } 100 \% \\
\text { Classificação: alta }(\geq 85 \%) \\
\text { ou baixa }(<85 \%) \text {. }\end{array}$ \\
\hline Estabilidade & $\begin{array}{l}\text { Número de vezes que o Hemocad ficou } \\
\text { inoperante em 2007. A quantidade de } \\
\text { profissionais entrevistados da VISA estadual foi } \\
\text { definida por conveniência }(\mathrm{N}=7) \text {. }\end{array}$ & $\begin{array}{l}\text { Pontuação: } 0 \text { a } 1 \\
\text { Classificação: alta (0) oubaixa (1). }\end{array}$ \\
\hline Oportunidade & $\begin{array}{l}\text { Rapidez do SH na(o): i) detecção e notificação de } \\
\text { eventos adversos pelos serviços de saúde; ii) } \\
\text { confirmação, definição do problema (surto, } \\
\text { tendência) e comunicação aos diferentes níveis de } \\
\text { gestão do sistema; e iii) planejamento de ações } \\
\text { preventivas e medidas de controle. }\end{array}$ & $\begin{array}{l}\text { Pontuação: } 0 \text { a } 12 \\
\text { Classificação: oportuno }(\geq 9) \\
\text { ou não oportuno }(<9) \text {. }\end{array}$ \\
\hline $\begin{array}{l}\text { Qualidade dos } \\
\text { dados }\end{array}$ & $\begin{array}{l}\text { Percentual de campos "não preenchidos ou com } \\
\text { informações não válidas" em seis variáveis do } \\
\text { relatório principal emitido pelo Hemocad. }\end{array}$ & $\begin{array}{l}\text { Pontuação: } 0 \text { a } 100 \% \\
\text { Classificação: excelente }(90 \%) \text {, } \\
\text { regular (70 a } 89 \%) \text { ou ruim }(< \\
70 \%) \text {. }\end{array}$ \\
\hline
\end{tabular}

ção de 0 a 100\% que classificou a efetividade em: excelente (> 90\%), ótima aceitável (76 a 90\%), regular $(46$ a $75 \%)$ ou baixa $(<45 \%)$. Para a interpretação do resultado da razão custo-efetividade foi utilizado, como teto de eficiência do Vigisan, o custo médio da atividade executada acrescido de $15 \%$ do seu valor. Nesta etapa do estudo foi adotada a perspectiva econômica da Anvisa.
Os dados obtidos foram organizados em bancos de dados no software Microsoft Excel $2003^{\circledR}$. Procedimentos de estatística descritiva foram utilizados para o cálculo de medidas de tendência central e de dispersão. Nas entrevistas foi disponibilizada uma carta de apresentação do estudo e solicitado o consentimento verbal dos profissionais. 

dependentemente da natureza jurídica, que desenvolva ações relacionadas com a gestão do sangue e de seus componentes, denominada de setor regulado, como hemocentros, núcleos de hemoterapia, unidade de coleta, unidade de coleta e transfusão e agência transfusional (Tabela 1). O foco é a proteção da saúde e, diferentemente de outros sistemas de vigilância em saúde pública, a atuação do Vigisan é pautada por um conjunto de normas vigentes no país. São exemplos de eventos sob VISA a transmissão de doenças pelo sangue, as reações transfusionais imediatas e tardias não infecciosas, o monitoramento da qualidade dos serviços de hemoterapia e de produtos sanguíneos disponibilizados para uso, bem como, toda e qualquer violação da legislação sobre sangue e seus componentes.

A coleta de informação se dá de forma passiva para quatro sistemas gerenciais de informação - Hemocad, Hemoprod (sistema nacional de vigilância de produção hemoterápica), AEQ-Sorologia e Imuno-hematologia e Sistema de Notificações em Vigilância Sanitária (Notivisa). Até meados de 2007, as ações relacionadas à vigilância do sangue eram coordenadas pela GESAC. Com a criação do Núcleo de Gestão do Sistema Nacional de Notificação e Investigação em Vigilância Sanitária (NUVIG), em 2005, as ações de hemovigilância foram incorporadas pela UBHEM.

Tabela 1. Número de serviços de hemoterapia, por nomenclatura estabelecida. Brasil, 2007.

\begin{tabular}{lrr}
\hline \multicolumn{1}{c}{ Serviço de hemoterapia } & \multicolumn{1}{c}{ N } & \multicolumn{1}{c}{$\%$} \\
\hline Hemocentro coordenador & 26 & 1,0 \\
Hemocentro regional & 65 & 3,0 \\
Núcleo de hemoterapia & 302 & 13,0 \\
Unidade de coleta e transfusão & 181 & 8,0 \\
Unidade de coleta & 13 & 0,5 \\
Central de triagem laboratorial & 13 & 0,5 \\
de doadores & & \\
Agência transfusional & 1.694 & 73,0 \\
Não informado & 18 & 0,8 \\
Total & $\mathbf{2 . 3 1 2}$ & $\mathbf{1 0 0 , 0}$ \\
\hline
\end{tabular}

Fonte: Hemocad/GESAC/GGSTO/Anvisa.

\section{Descrição das unidades de análise e dos componentes}

GESAC: essa unidade compõe, com outra gerência, a estrutura organizacional da GGSTO. Possui um gerente, seis técnicos de nível superior e uma secretaria para cumprir 15 atribuições. À exceção do Hemocad, os outros componentes avaliados estão explicitamente contemplados nas atribuições da GESAC. Esta, conta atualmente com dois sistemas de informações gerenciais em funcionamento, Hemocad e Hemoprod.

Normatização: o exercício de VISA na área de sangue é pautado na aplicação sistemática de um conjunto de normas criadas à luz de informações científicas. Essas normas, por sua vez, são elaboradas em circunstâncias nas quais os riscos à saúde da população são considerados inadmissíveis. São quatro as normas comumente utilizadas nas ações de VISA do sangue, a saber: i) Portaria $n^{\circ} 121$, de 24 de novembro de $1995^{13}$; ii) Lei no 10.205 , de 21 de março de $2001^{14}$; iii) Resolução no 151 , de 21 de agosto de $2001^{15}$; e iv) Resolução da Diretoria Colegiada (RDC) no 153 , de 14 de junho de $2004^{16}$. A não obediência à legislação sanitária federal implica em infrações previstas em lei ${ }^{17}$, as quais são punidas, alternativa ou cumulativamente, com várias penalidades.

Inspeção sanitária: este componente é fundamental para a VISA, pois a partir dele decorre uma série de ações que visam à redução e o controle do risco sanitário com o uso de sangue e de seus componentes. Não há nenhuma legislação sanitária federal que defina esta prática de VISA. A participação do nível federal nas inspeções sanitárias em serviços de hemoterapia somente ocorre quando por solicitação da VISA estadual. Para orientar as inspeções e minimizar as subjetividades dos inspetores são estabelecidos roteiros para a avaliação das unidades hemoterápicas. A inspeção em serviços de hemoterapia pode ser solicitada para a obtenção de licença de funcionamento ou renovação, monitoramento ou averiguação de denúncia pública. Em 2007 foram realizadas cinco inspeções que contaram com a participação de técnicos da GESAC.

AEQ: é uma avaliação interlaboratorial realizada a partir de testes de proficiência em imunohematologia e sorologia cujo objetivo é contribuir para o aumento da segurança transfusional por meio da garantia de qualidade e confiabilidade dos testes realizados pelos serviços de hemoterapia do país. Suas características são: educativo/não punitivo, confidencial, universal, oficial, adesão voluntária e com participação gra- 
tuita. O AEQ conta com dois sistemas de informação desenhados e desenvolvidos pela Anvisa, embora ainda em fase de consolidação.

A GGSTO, por meio de técnicos da GESAC, coordena o AEQ, que conta com nove instituições parceiras, denominadas de unidades produtoras, responsáveis pela produção e distribuição de painéis amostrais, sendo oito na área de imuno-hematologia e uma na de sorologia. O AEQ é assessorado por dois grupos técnicos formados por especialistas nas áreas de imuno-hematologia $(\mathrm{N}=8$ titulares) e de sorologia ( $\mathrm{N}=8$ titulares).

Hemocad: este sistema de informações gerenciais tem como principal meta o cadastramento de todos os serviços de hemoterapia do país. O Hemocad é formado por 29 tabelas próprias e sete auxiliares que se inter-relacionam para a finalização de um registro e a elaboração de relatórios. Para ter acesso ao sistema é necessário que o usuário esteja cadastrado no sistema de segurança da Anvisa. Conta com o manual do usuário versão de julho de 2001, no entanto, não apresenta um dicionário de variáveis. É um sistema web em que a base de dados é centralizada em um servidor na Anvisa. A alimentação dos dados no sistema é descentralizada e realizada pelas vigilâncias sanitárias estaduais.

O Hemocad conta com sete módulos de cadastramento e um para emissão de relatórios (15 tipos) previamente estabelecidos pelo gestor do sistema. Cada módulo de cadastramento possui variáveis a serem preenchidas tanto pelas vigilâncias estaduais quanto pela Anvisa. As vigilâncias estaduais visualizam apenas o módulo de "Cadastro de Serviços de Hemoterapia (CSH)" e "Relatórios". O CSH conta com 30 variáveis a serem preenchidas. Os outros módulos que compõem o sistema são tabelas acessórias que fornecem as opções de preenchimento do CSH, permitindo uma padronização do preenchimento. A periodicidade para a alimentação dos dados no sistema, a análise e a elaboração de relatórios não estão definidas pelo responsável e usuários do Hemocad, e no ano estudado não foi registrado nenhuma dessas atividades.

UBHEM: essa unidade foi criada em 2007 e compõe, juntamente com outras três áreas, a estrutura organizacional do NUVIG. Neste ano, possuía um chefe de unidade, um secretário, um técnico de nível superior na área administrativa e dois estagiários para cumprir 15 atribuições. Os eventos adversos de todo o país são registrados no Notivisa e gerenciados por essa unidade.

SH: esse sistema, em âmbito federal, é gerido pela UBHEM. Embora não exista um instrumen- to legal que crie e defina este sistema. Em documentos técnicos ${ }^{18,19}$ e no sítio da internet da Anvisa são feitas referências a um Sistema Nacional de Hemovigilância, o qual é definido como um sistema de monitoramento e alerta, organizado com o objetivo de coletar, avaliar e disseminar informações sobre os efeitos indesejáveis e/ou inesperados da utilização de sangue e componentes a fim de prevenir seu aparecimento ou recorrência.

O SH adota dois subsistemas de vigilância, o primeiro universal e passivo e o segundo sentinela e passivo. Este último conta com a participação de 210 hospitais que integram o projeto Hospitais Sentinela da Anvisa. A partir de 2007, a notificação passou a ser registrada no Notivisa, o qual aceita como fonte notificadora as instituições de saúde previamente cadastradas. Além disso, as informações são disponibilizadas à comunidade no sítio da internet da Anvisa, na forma de relatório.

Os eventos adversos passíveis de notificação incluem: reação hemolítica aguda e tardia, reação febril não hemolítica, reações alérgicas, sobrecarga volêmica, contaminação bacteriana, edema pulmonar não cardiogênico, reação hipotensiva e hemólise não imune, doenças transmissíveis (hepatites virais B e C, HIV/Aids, doença de Chagas, sífilis, malária), doença do enxerto contra o hospedeiro e aparecimentos de anticorpos irregulares/isoimunização ${ }^{18}$.

A investigação epidemiológica e sanitária da suspeita de transmissão de doenças pelo sangue e componentes pode ser desencadeada a partir da notificação recebida pelas vigilâncias epidemiológicas ou sanitárias. No entanto, a realização da investigação epidemiológica, segundo roteiros definidos em manuais técnicos ${ }^{18}$, não é feita diretamente pelo SH em âmbito federal. Nesses roteiros, o papel reservado para a Anvisa é de suporte técnico-administrativo.

\section{Avaliação geral}

Foram feitas $22(88 \%)$ entrevistas com profissionais das unidades de um total esperado de 25 , sendo nove do AEQ-Imuno-hematologia, quatro do AEQ-Sorologia, um do sistema de hemovigilância e oito para normatização e inspeção sanitária. Uma profissional respondeu a três questionários, totalizando 20 pessoas entrevistadas. Deste total, $75 \%(\mathrm{~N}=15)$ foram do sexo feminino. A mediana de idade $(\mathrm{N}=19)$ foi de 46 anos (intervalo: 28 - 56) e a mediana do tempo de atuação na VISA ( $\mathrm{N}=17)$ foi de 6 anos (intervalo: $2-27$ ). 
Na Tabela 2 encontra-se a avaliação geral do Vigisan quanto aos aspectos de estrutura, processo e resultados. Percebem-se problemas de estrutura e funcionamento, os quais foram avaliados como mal definida e regular, respectivamente.

Dentre os três componentes avaliados que compôs a avaliação geral, o SH foi o que apresentou menor pontuação na estrutura (mal definida), processo (funcionamento regular) e resultados (utilidade aceitável). A avaliação do aspecto "resultados" tanto do AEQ-Imuno-hematologia quanto do AEQ-Sorologia foi classificada como "muito útil". No entanto, os aspectos de estrutura e processo para o primeiro programa foram avaliados como: tendo imprecisões e deficiente, respectivamente.
A avaliação de desempenho do Vigisan está demonstrada no Gráfico 1. O indicador 4 conseguiu obter um desempenho "ótimo desejável" (75\%) enquanto que o indicador 2 apresentou o menor desempenho (45\%). Ademais, o valor médio de desempenho global dos quatro indicadores ficou também abaixo do "ótimo desejável" $(62 \%)$.

\section{Avaliação específica}

Simplicidade: o sistema foi classificado como complexo, pois os itens avaliados receberam seis pontos. Dos nove entrevistados que responderam aos quesitos, oito referiram ser complexa a forma de consolidar e analisar os dados do AEQImuno-hematologia; sete acharam complexa a

Tabela 2. Avaliação geral do Vigisan, segundo aspectos de estrutura, processo e resultados. Brasil, 2007.

\begin{tabular}{lccl}
\hline \multicolumn{1}{c}{ Aspectos } & Pontuação máxima & Pontuação alcançada* & \multicolumn{1}{c}{ Interpretação $^{*}$} \\
\hline Estrutura & 85 & 41 & Estrutura mal definida \\
Processo & 172 & 95 & Funcionamento regular \\
Resultados & 48 & 42 & Utilidade aceitável \\
\hline
\end{tabular}

${ }^{\star}$ Valores médios

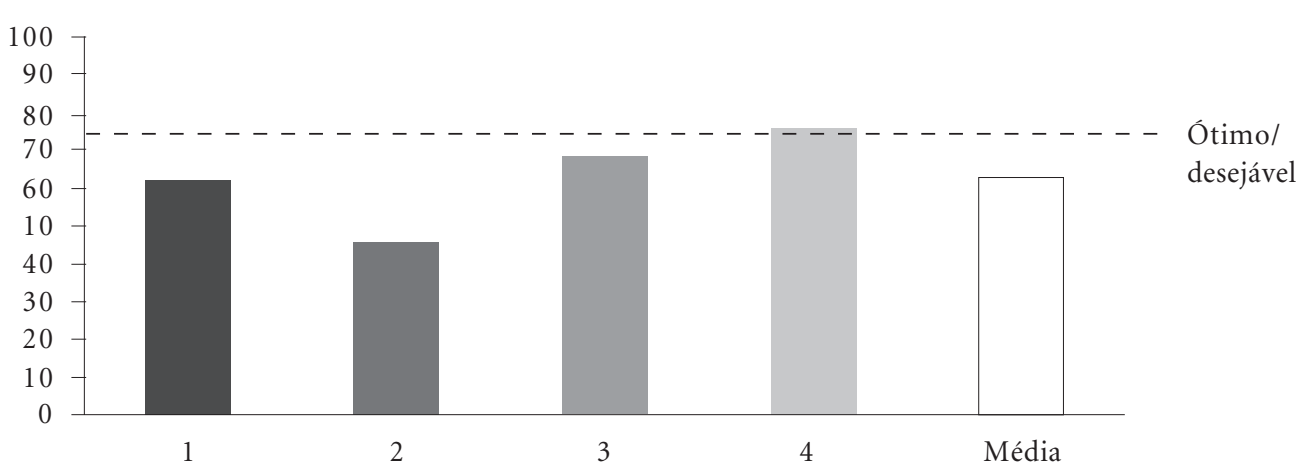

Indicadores

1. Revisão periódica, avaliação e modificação do marco regulatório

2. Capacidade técnica e institucional para fazer cumprir no âmbito do Estado as leis e normas relacionadas com o uso de sangue

3. Conhecimentos, habilidades e mecanismos para revisar, aperfeiçoar e fazer cumprir o marco regulatório

4. Cooperação e apoio técnico aos estados e municípios para inspeção sanitária e criação

de leis e normas relacionadas com o uso de sangue

Gráfico 1. Avaliação de desempenho do Vigisan. Brasil, 2007. 
forma de difundir e comunicar os dados; e este mesmo número de entrevistados considerou que os dados coletados não são analisados ou utilizados; o tempo gasto para a execução de tarefas do AEQ-Imuno-hematologia foi considerado como elevado, bem como a execução das tarefas é um aspecto que complica e afeta a dinâmica dessa avaliação interlaboratorial.

Flexibilidade: a transferência das atividades de hemovigilância não refletiu em custo adicional para o Vigisan quanto a incremento na previsão orçamentária de 2007. Este procedimento envolveu um repasse de recursos monetários previstos destinados a hemovigilância da planilha orçamentária da GESAC para o NUVIG.

A forma como o Vigisan respondeu ao novo problema "auto-hemoterapia" notificado à Anvisa foi por meio da publicação da Nota Técnica $n^{\circ} 1 / 2007^{20}$. O Vigisan teve a habilidade de adaptar-se às duas situações sem ocasionar mudanças significativas e sem produzir custos adicionais, sendo classificado como flexível.

Aceitabilidade: a proporção de hospitais sentinela que apresentaram planos de melhoria em hemovigilância foi de 30\% (31/104) o que classificou a aceitabilidade como baixa. Os hospitais da região Nordeste apresentaram a maior proporção de planos de melhoria (41\%).

Representatividade: um total de 19 (70\%) unidades da federação apresentou uma taxa percentual de representatividade $\geq 85 \%$, o que foi considerado suficiente para classificar o Vigisan com alta representatividade. As unidades federadas que não alcançaram uma pontuação superior ou igual a $85 \%$ foram: Alagoas, Goiás, Minas Gerais, Mato Grosso, Pará, Paraíba, Paraná e São Paulo, sendo que Goiás apresentou a menor representatividade (34\%). Em Roraima o número de serviços de hemoterapia cadastrados no Hemocad $(n=14)$ foi sete vezes superior àquele registrado no CNES $(n=2)$. No Hemocad, 89 (4\%) serviços de hemoterapia estavam com a situação de funcionamento definida como "fechada". Salienta-se que, anteriormente à obtenção dos dados para esta avaliação, os técnicos da GGSTO haviam realizado uma sensibilização com as vigilâncias sanitárias estaduais para a atualização do banco.

Estabilidade: a estabilidade foi considerada alta, pois dois dos seis técnicos entrevistados da vigilância sanitária estadual que utilizaram o Hemocad em 2007 citaram que o sistema ficou mais de uma vez inoperante (Goiás e Piauí). Em 2007, o Distrito Federal não fez uso do sistema.
Os outros estados pesquisados foram Bahia, Espírito Santo, Paraná e Rio Grande do Sul.

Oportunidade: os itens avaliados alcançaram uma pontuação de cinco, o que classificou o sistema como não oportuno. A confirmação, definição do problema (surto, tendência) e transmissão da informação aos diferentes níveis de gestão do SUS, bem como o planejamento de ações preventivas e medidas de controle foram referidos como demorados.

Qualidade dos dados: foram avaliados 14.412 campos, dos quais $2.020(14 \%)$ apresentaram algum tipo de problema no preenchimento da variável. Desta forma, $86 \%$ deles foram adequadamente preenchidos, o que classifica o Vigisan com qualidade de dados regular. As variáveis que não alcançaram um valor $\geq 90 \%$ foram: nível de complexidade $(87 \%)$, situação hemoterápica (84\%), responsável técnico $(69 \%)$ e código de endereçamento postal (83\%).

O Sistema apresentou problemas na estrutura e nos atributos simplicidade, aceitabilidade, oportunidade e qualidade dos dados, o que pode dificultar o cumprimento de seus objetivos e, consequentemente, sua utilidade. Em relação ao aspecto "resultado" a utilidade foi considerada como aceitável. Em dois dos três componentes analisados, o sistema foi avaliado como "muito útil”. Ademais, o Vigisan foi considerado como flexível, representativo e estável, qualidades que provavelmente o tornam mais útil para uma ação em saúde pública. Em relação à capacidade do sistema de, entre outros aspectos, detectar e notificar eventos adversos a sua utilidade foi considerada aceitável, porém pouco oportuna.

\section{Eficiência da operação do Sistema}

O custo total somado a outros componentes não avaliados foi superior a $\mathrm{R} \$ 3.500 .000,00$, sendo que o maior percentual foi registrado para o AEQ (32\%) seguido do SH (19\%). Os componentes avaliados, incluído o custo de pessoal, representaram $91,8 \%$ do custo total previsto no orçamento de 2007. O custo médio por atividade programada foi de R \$44.476,19 enquanto que para aquelas executadas este valor foi de $\mathrm{R} \$ 59.840,69$. A efetividade de $74 \%$ [(55 executadas/74 programadas $)^{\star} 100$ ] foi considerada como regular. A razão custo-efetividade foi de $\mathrm{R} \$ 80.865,80$ por atividade executada, ou seja, o sistema foi considerado ineficiente, pois a razão custo-efetividade foi superior ao valor de teto estabelecido ( $\mathrm{R} \$ 68.816,79)$. 


\section{Discussão}

Este estudo é o primeiro realizado no país que apresenta um panorama geral da qualidade da vigilância e eficiência do Vigisan a partir de três métodos avaliativos ${ }^{9-12}$. A intenção dos métodos de avaliação em saúde é desenvolver uma cultura avaliativa dos resultados na prática diária das organizações e dos profissionais, identificar os pontos fortes e os débeis de um sistema de vigilância visando o diagnóstico operacional de seus componentes e avaliar a eficiência da operação desse sistema e sua utilidade para a saúde pública. Essa cultura, no entanto, ainda não faz parte explicitamente da estratégia de gestão de muitos sistemas de vigilância em saúde pública.

No Brasil, os estudos publicados de avaliação de sistema de vigilância têm focalizado o campo da vigilância epidemiológica e utilizado a metodologia desenvolvida pelo CDC/EUA ${ }^{21-23}$ que prioriza as atividades típicas de vigilância como notificação de casos, investigação epidemiológica e respostas aos surtos. Segundo Silva Junior ${ }^{24}$, essa metodologia, no entanto, não cobre aspectos operacionais e gerenciais importantes, além de não ser dirigida para uma avaliação que sintetize o conjunto das ações executadas em uma determinada área.

No caso da avaliação de sistemas de VISA, são escassos os trabalhos publicados no Brasil e em outros países, o que dificultou a discussão dos resultados obtidos. Um estudo encontrado na literatura foi realizado por Battesini ${ }^{8}$, que propôs um método multidimensional para avaliação de desempenho da VISA. O método foi aplicado, também, em nível municipal, permitindo avaliar a implantação da VISA em relação ao entendimento de técnicos, gestores e conselheiros de saúde. A avaliação apontou um desempenho global regular, indicando a necessidade de adequação nas ações de VISA. Este autor cita ainda que os estudos de avaliação de sistemas de VISA podem auxiliar na superação de fragilidades presentes na avaliação normativa (Termos de Ajustes e Metas e Programações Pactuadas) e naquelas subjetivamente embasadas em discursos de governo ${ }^{8}$.

Uma das limitações deste estudo está relacionada à possibilidade de viés de aferição dos resultados, pois parte da coleta de dados ocorreu de forma subjetiva, somado ao fato dos entrevistados terem avaliado o serviço no qual os mesmos estão inseridos, o que poderia ocasionar numa visão parcial da realidade. Desta forma, o viés estaria sendo produzido pela própria fonte de da- $\operatorname{dos}^{25}$. Ademais, parte dos resultados foi obtida por informações referidas, o que limita a possibilidade de comparações ao longo do tempo.

Outras limitações foram: i) não avaliação dos atributos, sensibilidade e valor preditivo positivo, preconizados pela metodologia do CDC/ EUA; ii) adesão de consultores do AEQ-Sorologia não foi completa; iii) o valor financeiro da previsão orçamentária de janeiro de 2007 foi utilizado para o cálculo da razão custo-efetividade; e iv) os custos com pessoal dedicado aos componentes avaliados estão superestimados, pois o tempo a outras atividades desenvolvidas pelos profissionais foi incluído na sua contabilização.

Handler et al. ${ }^{26}$ propõem o uso da tríade estrutura-processo-resultado para a avaliação de desempenho de sistemas públicos de saúde. A avaliação geral do Vigisan quanto aos aspectos estudados apontou, principalmente, para problemas de estrutura. De acordo com Battesini ${ }^{8}$, não é possível imaginar um desempenho satisfatório da VISA sem uma estrutura adequada que lhe dê suporte para responder as inúmeras demandas sociais e àquelas previstas na Constituição Federal.

Parte da responsabilidade de gestores da VISA está relacionada com a elaboração e o cumprimento das normas ${ }^{27}$. Estas ações devem ser encaradas como um serviço de promoção e proteção da saúde, uma vez que são aplicadas em situações de fragilidade e de ameaça à saúde. Neste estudo, o desempenho do Vigisan apresentou resultados inferiores ao esperado. Medidas para a ampliação dos esforços na melhoria dos componentes de normatização e inspeção sanitária devem ser adotadas, pois são elementos primordiais para a realização das práticas de VISA e que melhor identificam essa vigilância nos três níveis de gestão do SUS.

Dos sete atributos estudados três alcançaram resultados positivos: flexibilidade, representatividade e estabilidade. Os esforços para melhorar os atributos simplicidade, aceitabilidade, oportunidade e qualidade dos dados podem favorecer um aumento na utilidade do sistema, tornando-o mais completo. No entanto, os diversos atributos que compõem a avaliação de um sistema de vigilância são interdependentes e o aprimoramento de um pode influenciar no desempenho de outro ${ }^{12,28}$. Desta forma, não se deve avaliar separadamente esses atributos, mas sim, ter uma preocupação com um equilíbrio apropriado entre eles ${ }^{28}$.

A simplicidade de um sistema de vigilância em saúde pública diz respeito à sua estrutura e à 
facilidade de operação ${ }^{12,28}$. Neste estudo, o Vigisan, baseado no componente AEQ imuno-hematologia, foi considerado complexo estando diretamente relacionado com aspectos para operar o sistema. A aceitabilidade reflete a vontade de pessoas e instituições em participar do sistema de vigilância. A baixa aceitabilidade na elaboração de planos de melhoria em hemovigilância por hospitais que compõem o Projeto Hospitais Sentinela reflete na quantidade de notificações de eventos adversos relacionados com sangue e componentes registrada no Notivisa.

Segundo a metodologia do $\mathrm{CDC}^{12}$ deve ser avaliada a oportunidade de um sistema de vigilância em termos de disponibilidade de informação para controle de um evento sanitário. A necessidade para rapidez de resposta em um sistema de vigilância dependerá da natureza do evento sanitário sob vigilância e dos objetivos do sistema. A esse atributo, o Vigisan foi considerado não oportuno, pois a velocidade entre diversos passos importantes para o controle e a minimização do risco foi referida como problemática.

A qualidade dos dados reflete a completitude e a validade dos dados registrados no sistema de vigilância em saúde pública ${ }^{12,28}$. Neste estudo, embora o sistema tenha alta representatividade, essa condição não refletiu na qualidade dos dados, pois este atributo foi avaliado como regular. Em geral, sistemas ativos de vigilância obtêm dados de melhor qualidade comparados aos obtidos pelos sistemas passivos ${ }^{28}$.

Santos e Garrett, ao avaliarem o sistema de vigilância de hantavírus no Brasil, evidenciaram que o mesmo é complexo, pouco flexível, instável, com baixa aceitabilidade e representatividade, além da insatisfatória qualidade dos dados. Ademais, o sistema carece de grandes quantidades de recursos. Entretanto, mostrou ser de grande utilidade para detectar casos de síndrome cardiopulmonar por hantavírus e identificar surtos, orientar medidas de prevenção e controle e gerar mudanças nas práticas clínicas e de vigilância ${ }^{21}$.

Embora a utilidade do Vigisan tenha sido considerada aceitável, a estrutura e o funcionamento apresentaram problemas que podem comprometer os resultados do sistema, incluído o cumprimento de seus objetivos. O componente $\mathrm{SH}$ deve ser priorizado quanto a esses aspectos, o que provavelmente aumentaria a oportunidade das suas ações de vigilância.
Faz-se necessário reavaliar o funcionamento do AEQ-Imuno-hematologia em relação à gestão da informação, principalmente quanto à forma de consolidar e analisar os dados, definir um método para análise periódica, o período de análise, as variáveis a serem incluídas e a forma de retroalimentação.

Quanto à eficiência na operação do Vigisan, é aconselhável garantir que um maior número de atividades programadas seja executado com vistas a melhorar a efetividade e, consequentemente, a reduzir a perda de eficiência para o sistema. Esse objetivo visa atender aos princípios da Administração Pública discriminados na Constituição Federal ${ }^{7}$, particularmente, o princípio da eficiência - alcançar os melhores benefícios a menor custo possível.

\section{Conclusões}

As deficiências apontadas neste estudo devem ser uma desencadeadora de ações para modificar ou até mesmo excluir componentes que não cumprem com sua finalidade enquanto instrumento de fortalecimento dos objetivos do sistema. Esses problemas identificados, e que influenciam a qualidade do sistema, podem ter contribuído com a avaliação não tão positiva da eficiência do Vigisan. Apesar disso, o sistema foi considerado útil para a saúde pública do país, encontra-se implantado na Anvisa e tem contribuído em garantir um suprimento seguro e adequado de sangue e de seus componentes para a população brasileira. Diversas atividades podem ser desenvolvidas para a superação dessas deficiências como o planejamento integrado das ações entre as unidades da Anvisa, aperfeiçoamento e expansão do SH, bem como de outros componentes avaliados e a produção e o uso de informações epidemiológicas como insumo estratégico para as ações de vigilância sanitária do sangue em âmbito federal.

Este estudo apontou ainda para a importância da realização de avaliações de Sistemas de Vigilância em Saúde Pública, as quais podem ser vistas como fundamentais e complementares à avaliação normativa prevista em contratos de gestão acordados entre os entes que compõem o SNVS. 


\section{Colaboradores}

DM Mota foi responsável pelo planejamento e execução do estudo e redação do manuscrito. DRC Freitas e WN Araújo participaram do planejamento e execução do estudo e da revisão final do texto.

\section{Referências}

1. Organização Mundial da Saúde (OMS). Transfusão de Sangue Seguro. 1999. [acessado 2008 mai 26]. Disponível em: http://www.who.int/bloodsafety/ transfusion_services/en/Blood_Safety

2. Stainsby D, Russell J, Cohen H, Lilleyman J. Reducing adverse events in blood transfusion. $\mathrm{Br} J$ Haematol 2005; 131(1):8-12.

3. Schreiber GB, Busch MP, Kleinman SH, Korelitz JJ. The risk of transfusion-transmitted viral infections. N Engl J Med 1996; 334(26):1685-1690.

4. Agência Nacional de Vigilância Sanitária (Anvisa). Boletim de Hemovigilância 2008. [acessado em 2010 jul 21]. Disponível em: http://www.anvisa.gov.br/ sangue/hemovigilancia/index.htm.

5. Schmunis GA, Cruz JR. Safety of the blood supply in Latin America. Clin Microbiol Rev 2005; 18(1):12-29.

6. Costa EA Vigilância sanitária: proteção e defesa da saúde. $2^{\text {a }}$ ed. São Paulo: Sociedade Brasileira de Vigilância de Medicamentos. 2004.

7. Brasil. Presidência da República. Casa Civil. Constituição da República Federativa do Brasil de 1988. [acessado 2010 jan 19]. Disponível em: http://www. planalto.gov.br/ccivil_03/constituicao/constitui\% C3\%A7ao.htm.

8. Battesini M. Método multidimensional para avaliação de desempenho da vigilância sanitária: uma aplicação em nível municipal [tese]. Porto Alegre: Universidade Federal do Rio Grande do Sul; 2008.

9. Moliner RB, Ochoa EG. Evaluación de la vigilância em la atención primaria de salud: una propuesta metodológica. Rev Cubana Med Trop 2000; 52(1):5565.

10. Organização Pan-Americana da Saúde (OPAS). A saúde pública nas Américas. Instrumento para a medição do desempenho das funções essenciais de saúde pública. Washington: Organização Pan-Americana da Saúde (OPAS); 2001.

11. Organização Pan-Americana da Saúde (OPAS). Medição do desempenho das funções essenciais de saúde pública. Guia para a aplicação do instrumento de desempenho das funções essenciais de saúde pública. Washington: Organização Pan-Americana da Saúde (OPAS); 2001

12. Centers for Disease Control. Guidelines for evaluating surveillane systems. Morb.Mort.wkly Rep. 1998; 37(5S):1-18

13. Brasil. Ministério da Saúde (MS). Portaria no 121 de 24 de novembro de 1995. Institui como norma de inspeção para órgãos de vigilância sanitária do Sistema Único de Saúde, o "Roteiro para Inspeção em Unidades Hemoterápicas. Diário Oficial da União 1995; 30 nov.

14. Brasil. Lei 10.205 de 21 de março de 2001. Regulamenta o $\$ 4^{\circ}$ do art. 199 da Constituição Federal. Diário Oficial da União 2001; 21 mar.

15. Agência Nacional de Vigilância Sanitária (Anvisa). Resolução da Diretoria Colegiada da Anvisa no 151 de 21 de agosto de 2001. Regulamento Técnico sobre Níveis de Complexidade dos Serviços de Hemoterapia, que consta como anexo. Diário Oficial da União 2001; 22 ago. 
16. Agência Nacional de Vigilância Sanitária (Anvisa). Resolução da Diretoria Colegiada da Anvisa no 153 de 14 de junho de 2004. Determina o Regulamento Técnico para os procedimentos hemoterápicos. Diário Oficial da União 2004; 24 jun.

17. Brasil. Lei 6.437 de 20 de agosto de 1977. Configura infrações à legislação sanitária federal, estabelece as sanções respectivas, e dá outras providências. Diário Oficial da União 1997; 24 ago.

18. Agência Nacional de Vigilância Sanitária (Anvisa). Hemovigilância: manual técnico para investigação das reações transfusionais imediatas e tardias não infecciosas. Brasília: Agência Nacional de Vigilância Sanitária (Anvisa); 2007.

19. Agência Nacional de Vigilância Sanitária (Anvisa). Relatório do Sistema Nacional de Hemovigilância 2002 a 2005. Brasília: Agência Nacional de Vigilância Sanitária (Anvisa); 2007. [acessado 2008 jun 30]. Disponível em: http://www.anvisa.gov.br/sangue/ hemovigilancia/relatorio_2002_2005.pdf

20. Agência Nacional de Vigilância Sanitária (Anvisa). Nota Técnica $n^{\circ} 1$ de 13 de abril de 2007. [acessado 2008 maio 26]. Disponível em: http:// www.anvisa.gov.br/sangue/informes/

21. Santos ED, Garrett DO. Avaliação do Sistema de Vigilância de Hantavírus no Brasil. Epidemiologia e Serviços de Saúde 2005; 14(1):15-31.

22. Menezes MJR. Avaliação do Sistema de Vigilância Epidemiológica da Esquistossomose no Estado da Bahia [dissertação]. Rio de Janeiro: Fundação Oswaldo Cruz (Fiocruz), Escola Nacional de Saúde Pública (ENSP); 2005

23. Madeira A, Lanzieri TM, Paz LC. Avaliação da notificação da sífilis congênita no Brasil, 2000 a 2003. Boletim eletrônico epidemiológico 2007; 7(7):1-5.

24. Silva-Júnior JB. Epidemiologia em serviço: uma avaliação de desempenho do Sistema Nacional de Vigilância em Saúde [tese]. Campinas: Universidade Estadual de Campinas (Unicamp); 2004.

25. Souza JS, Stein AT. Vigilância sanitária de uma cidade metropolitana do sul do Brasil: implementação da gestão plena e efetividade das ações. Cien Saude Colet 2008; 13(Supl. 2):2225-2238.

26. Handler A, Issel M, Turnock B. A conceptual framework to measure performance of public health system. Am J Public Health 2001; 91(8):1235-1239.

27. Villalbí JR, Cusí M, Caylá JÁ, Duràn J, Guix J El ejercicio de La autoridad sanitária: los princípios, lo reglamentado y La incertidumbre. Gac. Sanit. 2007; 21(2):172-175.

28. Waldman EA. Vigilância como prática de Saúde Pública. In: Campos GWS, Minayo MCS, Akerman M, Drumond-Júnior M, Carvalho YM, organizadores. Tratado de Saúde Coletiva. São Paulo: Hucitec, Fundação Oswaldo Cruz (Fiocruz); 2006. p. 487-528. 